\title{
Criminologie
}

\section{L'homicide conjugal à Montréal, ses raisons, ses conditions et son déroulement}

\section{Maurice Cusson et Raymonde Boisvert}

Volume 27, numéro 2, 1994

Sexe et criminalité

URI : https://id.erudit.org/iderudit/017359ar

DOI : https://doi.org/10.7202/017359ar

Aller au sommaire du numéro

\section{Éditeur(s)}

Les Presses de l'Université de Montréal

ISSN

0316-0041 (imprimé)

1492-1367 (numérique)

Découvrir la revue

Citer cet article

Cusson, M. \& Boisvert, R. (1994). L’homicide conjugal à Montréal, ses raisons, ses conditions et son déroulement. Criminologie, 27(2), 165-183.

https://doi.org/10.7202/017359ar
Résumé de l'article

Conjugal homicide is a situation where one person murders another person with whom he or she is involved through a matrimonial, quasi-matrimonial or other romantic relationship, the study of this type of homicide is based on the entirety of conjugal murders known to police (77) and committed in different municipalities on the island of Montreal during two time periods, namely 1954 to 1962 and 1985 to 1989 . The great majority of these crimes are committed by a man onto a woman. Analyses show that possessiveness - understood to be the desire of one person to exclusively control the other - is by far the reason which leads a man to murder the woman he supposedly loves. However, this desire to possess or control is not in itself sufficient for a man to execute his criminal activity, since a number of conditions must coexist : the woman questions her relationship with the man ; the man may physically strike the woman ; the man has the advantage of greater physical strength; the period of time involved is sufficiently lengthy allowing the crisis to develop and enter its critical phase and finally, the perpetrator succeeds in surpassing the inhibitions which initially impede one from killing another. 
Conjugal homicide is a situation where one person murders another person with whom he or she is involved through a matrimonial. quasi-matrimonial or other romantic relationship. the study of this type of homicide is based on the entirety of conjugal murders known to police (77) and committed in different municipalities on the island of Montreal during two time periods, namely 1954 to 1962 and 1985 to 1989. The great majority of these crimes are committed by a man onto a woman. Analyses show that possessiveness - understood to be the desire of one person to exclusively control the other - is by far the reason which leads a man to murder the woman he supposedly loves. However. this desire to possess or control is nor in itself sufficient for a man to execute his criminal activity, since a number of conditions must coexist: the woman questions her relationship with the man; the man may physically strike the woman; the man has the advantage of greater physical strength; the period of time involved is sufficiently lengthy allowing the crisis to develop and enter its critical phase and finally, the perpetrator succeeds in surpassing the inhibitions which initially impede one from killing another.

Dans le présent article, un homicide sera qualifié de conjugal quand un individu a tué une personne avec qui il était uni par un lien matrimonial, quasi matrimonial ou amoureux. Sur l'île de Montréal, il se commettait, entre 1985 et 1989 . environ 10 homicides conjugaux par année. Durant cette courte période. nous ne pouvons déceler de tendance ni à la hausse ni à la baisse. Les statistiques des dernières années nous autorisent à penser que cette relative stabilité se maintient jusqu'à aujourd'hui (Gouvernement du Québec. 1993).

Ce type d'homicide représente la forme ultime de violence conjugale. Selon Larouche (1987, p. 58), il est généralement le dernier échelon de la violence entendue sous toutes ses tormes : psychologique, morale, verbale, sexuelle et physique. Cette violence mortelle est aussi celle sur laquelle nous disposons des informations les plus nombreuses. En effet, ce crime ne

I. Dirceteur de l'École de criminologie et chercheur au Centre international de criminologic comparéc a l Université de Montréal, C.P. 6128, succ. Centre-ville, Montréal. QC H3C 3J7

2. Assistante de recherche, Centre international de criminologic comparée 
reste pratiquement jamais inconnu; il déclenche une enquête policière sérieuse et, bien souvent, les journalistes décrivent le drame avec un luxe de détails. L'information disponible sur l'homicide conjugal est sans commune mesure avec celle que nous avons sur les événements touchant les femmes violentées. Curieusement, on trouve peu d'analyses systématiques de l'homicide conjugal dans les ouvrages consacrés à la violence faite aux femmes. Compte tenu de la parenté entre ces deux crimes, il est probable que l'étude de l'homicide conjugal ajoute un élément à la compréhension du phénomène plus général de la violence au sein des couples.

\section{L'ÉTAT DE LA QUESTION}

Les auteurs s'entendent sur le fait que (sauf aux États-Unis) l'homicide conjugal est commis la plupart du temps par un homme sur une femme (Zhan. 1975: Chimbos, 1978: Dobash et Dobash, 1979; Daly, Wilson et Weghorst, 1982: Daly et Wilson. 1988a: Goetting. 1989; Stout, 1989; Polk et Ranson. 1991: Dobash. Dobash, Wilson et Daly, 1992 ; Wilson et Daly, 1992; Silverman et Kennedy, 1993).

Les données de la présente analyse vont dans ce sens : sur les 77 homicides conjugaux répertoriés dans l’île de Montréal 27 commis entre 19541962 et 50 entre $1985-1989)$, il s'en trouve $66(85.7 \%)$ qui sont le fait d'un homme tuant sa conjointe ou sa maîtresse. $4(5,2 \%)$ qui sont exécutés par un homme sur son amant et $7(9,1 \%)$ qui sont commis par une femme sur son compagnon. La question qui surgit à l'esprit est donc : «Pourquoi un homme en arrive-t-il à tuer sa compagne ou la femme qu'il prétend aimer?»

Deux genres de réponses peuvent être proposées à cette question. Les premières peuvent être qualifiées de causales. Par exemple, dans une étude transculturelle. Gartner (1990) montre que les taux d'homicides ayant une femme pour victime (qui sont majoritairement des homicides conjugaux) varient en raison directe du taux de divorce et de l'indice Gini d'inégalité ${ }^{3}$. Cette recherche et quelques autres tendent à montrer que l'instabilité des couples et les difficultés économiques sont des facteurs ou des «causes» de l'homicide conjugal (Chimbos. 1978; Blau et Blau, 1982; Messner. 1982; Zimring. Mukherjee et Van Winkle, 1983; Williams, 1984 : Parker et Toth, 1990).

Les réponses du second genre sont résolument non-causales. Il s'agit moins d'expliquer que de comprendre. Plutôt que de repérer les déterminismes

3. L'indice Gini mesure la différence moyenne entre les familles par rapport au revenu moyen. La valeur de l'indice Gini varic entre 0 et 1 el la valcur la plus élevéc signilic une plus grande inégalité dans la distribution des revenus. 
plus ou moins lointains qui pèsent sur le criminel, l'on recherche le sens des gestes posés par les acteurs du drame familial, en s'interrogeant sur leurs raisons et sur les circonstances qui pourraient l'éclairer. Cette idée que les phénomènes sociaux procèdent de décisions d'acteurs en situation qui poursuivent des buts s'affirme comme un paradigme majeur en sociologie (Boudon, 1992). En criminologie. elle jette un éclairage nouveau sur plusieurs délits, incluant les crimes de violence (Cornish et Clarke. 1986: Cusson, 1981: Cusson et Cordeau, 1993: Felson, 1993). Appliquée à l'homicide conjugal. cette problématique incite le chercheur à poser trois questions: 1) Quelles raisons ont poussé le meurtrier à poser le geste fatal? 2) Quelles conditions ont rendu le crime possible? 3) Au terme de quel concours de circonstances l'homicide a-t-il été perpétré? Reprenons ces trois points.

1. Le premier moment d'une démarche visant à comprendre l'homicide conjugal consiste à s'interroger sur les fins que poursuivait son auteur et sur le problème qu'il a voulu ainsi résoudre.

Selon les auteurs qui ont étudié la violence conjugałe en général. la fin de l'agresseur est la domination et le problème qu'il prétend résoudre est de l'ordre du contrôle. Les épisodes de violence s'inscrivent dans des rapports de domination. La brutalité est le fait d'hommes qui ont appris à attendre de leur compagne la soumission. Il y ont recours pour obtenir, par la force, ce qui leur apparaît comme un droit ou en réaction à ce qu'ils considèrent comme l'insoumission de la femme (Martin, 1976; Dobash et Dobash, 1979, 1988: Berk et al., 1983; Saunders, 1983; Martin, 1988: Dobash et al. 1992).

S'agissant de l'homicide conjugal, les principaux auteurs qui se sont intéressés aux raisons s'entendent pour accorder une importance déterminante à la jalousie masculine (Proal, 1900; De Greeff, 1942; Rabinowicz. 1931: Guttmacher, 1955; Chimbos, 1978; Nettler, 1982: Guillais, 1986: Daly et Wilson, 1988). La jalousie est ici conçue dans un sens large: la volonté masculine de possession exclusive de l'autre. Concrètement, cela signifie que l'homme tue sa conjointe parce qu'il refuse qu'elle le quitte ou qu'elle en aime un autre. Il croit avoir des droits sur elle, sur son amour, sur sa vie. Le meurtre est un acte commis pour se venger de l'abandon et pour empêcher la femme de se donner à qui que ce soit d'autre. Il exprime le refus total de laisser partir celle qu'il croit posséder de manière exclusive. C'est pourquoi, plutôt que de parler de jalousie, vaudrait-il mieux parler de possession s'inscrivant dans un rapport de domination.

Quelques chercheurs ont tenté de chiffrer la proportion des cas de jalousie parmi l'ensemble des homicides conjugaux. Par exemple, au Canada. entre 1974 et 1983 , la police a attribuế $21.3 \%$ des meurtres conjugaux à la jalousie (Daly et Wilson, 1988, p. 200)). Cependant, selon ces auteurs, les rapports de police ainsi que les statistiques sous-estiment l'importance de la 
jalousie. La présence d'une altercation masque souvent ce caractère de possession, de domination. Quand le chercheur interroge les meurtriers eux-mêmes sur la source du conflit qui a culminé jusqu'au meurtre, les pourcentages de cas de jalousie sont beaucoup plus élevés que ceux qui sont calculés par les chercheurs à partir des rapports de police. Par exemple. Chimbos (1978), qui avait interrogé 34 responsables de la mort de la personne qui partageait leur vie ( 29 hommes et 5 femmes). conclut que la jalousie sexuelle masculine dominait dans $85 \%$ de ces cas. La difficulté que soulèvent de tels chiffres est que la jalousie dominatrice n'apparaît pas toujours: les altercations, la rage et, à l'occasion. les disputes sur des questions d'argent viennent s'y greffer. Le premier objectif de cet article est d'établir, à partir des informations que nous trouvons dans les dossiers et les journaux, la proportion des homicides conjugaux dans lesquels la possession a un rôle à jouer pour ensuite illustrer le rôle qu'elle joue par le récit de quelques affaires typiques.

2. L'examen des circonstances et des conditions qui ont rendu possible le crime conjugal nous fait faire un pas de plus dans la compréhension du phénomène. Un mari peut imaginer avoir toutes sortes de raisons de vouloir tuer son épouse et ne pas le faire parce que les circonstances l'en empêchent. S'il finit par l'abattre, c'est non seulement parce qu'il le voulait, mais encore, parce qu'il a pu réunir les conditions nécessaires à son exécution.

Depuis la formule célèbre de Cohen et Felson (1979), selon qui, pour qu'un crime «prédateur» existe, il faut qu'un délinquant motivé, une cible intéressante et l'absence de gardien convergent dans un lieu et à un moment donne, les criminologues ont poursuivi une réflexion sur les conditions nécessaires du crime. Gottfredson et Hirschi (1990, p. 33) parlent à ce propos de la «structure logique» du crime. S'agissant de l'homicide (en général), ils affirment que cette structure comporte cinq éléments : 1) le criminel et la victime sont en interaction l'un avec l'autre; 2) le criminel dispose du moyen de tuer l'autre; 3 ) le meurtrier a une maîtrise de soi insuffisante pour l'empêcher de passer à l'acte: 4) la victime ne peut pas se retirer de la situation dangereuse dans laquelle elle se trouve: 5) il ne se trouve pas sur les lieux de protecteur qui pourrait sauver la vie de la victime.

Cette réflexion sur les conditions nécessaires à l'accomplissement d'un crime n'a pas été poursuivie à propos de l'homicide conjugal. Nous nous proposons, comme deuxième objectif, d'apporter une contribution aux connaissances sur ce type d'homicide.

3. Pour le commun des mortels, un moyen de faire comprendre le sens d'une action est de faire le récit des événements qui l'ont précédé. C'est aussi la démarche de l'historien. Pour ce dernier. le récit des faits est déjà 
un début d'explication. L'homicide conjugal est un conflit qui s'inscrit dans l'histoire d'une relation entre deux personnes et. le plus souvent, il a été précédé d'échanges très vif́s au cours desquels les paroles et les gestes de l'un répondent à ceux de l'autre pour ensuite susciter une riposte.

Le déroulement de l'homicide conjugal a été peu étudié. De Greeff (1942) avait bien décrit les étapes conduisant à l'acte fatal : l'assentiment inefficace, l'assentiment formulé et la crise, mais cela décrit le cheminement intérieur du meurtrier et non les événements. Plus récemment. Luckenbill (1977) a montré l'importance de l'enchaînement des faits lors de l'homicide interpersonnel typique. Selon lui, il est possible d'identifier cinq étapes dans un conflit qui se termine par la mort de l'un des deux protagonistes. 1) La victime refuse d'optempérer et l'agresseur interprète ce refus comme une offense personnelle; 2) il réplique par des menaces verbales ou physiques: 3 ) la victime répond par la provocation ou par les coups; 4) les échanges physiques s'ensuivent et les armes (ou ce qui tombe sous la main) viennent aggraver l'affrontement : 5) un des deux adversaires. emporté par la colère, la haine ou l'orgueil porte le coup fatal.

Le troisième et dernier objectif de cet article est de décrire les étapes du déroulement d'un homicide conjugal.

\section{LA MÉTHODOLOGIE}

Les données sur les homicides commis sur l'île de Montréal ont été recueillies à plusieurs sources qui se complètent les unes les autres: le Centre de pré-archivage du ministère de la Justice du Québec, les dossiers de la Cour supếrieure du palais de justice de Montréal et le journal Allô Police.

Pour identifier les affaires de meurtres, le plumitif de la Cour était sans intérêt. Nous avons donc utilisé l'hebdomadaire montréalais de langue française Allô Police, un journal spécialisé dans les homicides qui s'est avéré la seule source valable qui permettait de distinguer rapidement les homicides conjugaux des autres types à partir de la relation entre les protagonistes. En effet, depuis février 1953, une fois par année. le journal publie toutes les affaires de meurtres qui se sont déroulées au Québec durant l'année précédente.

Nous sommes partis de ce bilan annuel publié par Allô Police et nous avons repéré la totalité des homicides perpétrés sur l'île de Montréal entre 1954-1962 puis entre 1985-1989. Nous avons vérifié le caractère exhaustif du bilan annuel en repassant tous les numéros du journal pour les années concernées. Cette base de données a été confrontée systématiquement avec celle publiée par statistique Canada et nous a convaincus de la tiabilité de la première (Grenier, 1993). Nous avons ainsi répertorié un total de 511 homi- 
cides (149 exécutés durant la première période et 362 durant la seconde). Les affaires de négligence criminelle ont été éliminées de l'étude. En classant les homicides selon leur nature, nous en avons relevés 77 commis au détriment d'un-e conjoint-e, marié-e ou non, d'un-e ex-conjoint-e ou d'un-e amant-e (27 entre 1954 à 1962 et 50 entre 1985 à 1989). Il ne s'agit donc pas d'échantillons mais de populations complètes d'homicides conjugaux connus et enregistrés ${ }^{4}$ durant deux périodes contrastées.

Ces deux époques séparées par un intervalle de 23 ans ont été choisies pour obtenir un effet de contraste entre deux périodes fort différentes de notre histoire récente. Premièrement. les taux d'homicides se répartissent différemment : selon le Bureau fédéral de la statistique, le taux en 1964 se situait a 1,5 par 100000 habitants et à 2,51 en 1989. Ensuite, nous sommes confrontés à des différences, bien connues, entre les deux époques tant sur la plan politique, social que culturel. Les neuf années comprises dans la période avant la Révolution tranquille, soit celle allant de 1954 a 1962 , apparaissent comme étant relativement paisibles. Quant aux années 1985 à 1989, elles sont considérées comme la «période contemporaine»: elle est très proche de nous et rien n'indique qu'il y a eu des changements majeurs en matière d'homicides au Québec depuis 1989 (Statistique Canada, 1992). De plus, la cueillette des données devait se terminer en 1989, car nous voulions étudier des affaires terminées à la Cour. Nous avons décidé d'inclure deux périodes d'inégale durée dans l'étude afin d'éviter que les nombres d'homicides soient trop disproportionnés les uns par rapport aux autres.

Des formulaires très détaillés ont été préparés pour la cueillette des données qui devaient être enregistrées et codées pour permettre un traitement statistique. Nous ne nous sommes pas contentés de codifier les informations qui s'y prêtaient. Nous avons voulu recueillir le maximum de renseignements qualitatifs pour pouvoir ensuite faire le récit des événements et, si possible, comprendre la signification du drame. Une partie est prévue à cette grille d'analyse pour y inscrire les informations de nature qualitative sur lesquelles s'appuie la majeure partie de ce texte.

Nous avons d'abord dépouillé les dossiers déposés au Centre de préarchivage du ministère de la Justice du Québec pour les années 19541962 et ceux de la Cour supérieure du palais de justice de Montréal pour les années 1985 à 1989 (l'accès aux dossiers des procureurs et à ceux de la police, en regard de ces dernières années, a été refusé). Par la suite. tous les numéros du journal ont été soigneusement feuilletés pour compléter (ou recueillir dans les cas où il n'y avait pas de dossier) les données quantitatives et qualitatives. Pour les deux époques réunies, 61 dossiers criminels

4. Une sous-estimation est toujours possible, car il peut arriver que certains meurtres aient passé pour des décès naturels. 
ont été consultés ( 14 meurtriers s'étaient suicidés et deux autres dossiers étaient manquants). Nous disposons, dans la plupart des cas, de descriptions assez précises permettant d'identifier les raisons, les conditions et le déroulement de l'événement meurtrier.

\section{Les raisons de l'homicide conjugal}

Dans quel but et pour résoudre quel problème l'homicide conjugal est-il commis? Compte tenu de l'importance accordée à la jalousie masculine et au désir de possession sexuelle exclusive par la plupart des auteurs qui se sont posé cette question, nous avons classé dans les catégories «possession" tous les cas où ce problème se posait même si d'autres raisons pouvaient simultanément intervenir. Opérationnellement. tombaient dans cette classe toutes les affaires où le geste fatal est une réponse à l'annonce d'une rupture. à une rupture consommée (départ, séparation. divorce) ou à une liaison avec un rival. À partir des informations que nous trouvions dans les dossiers et dans les journaux et aussi, de notre lecture du mobile le plus apparent. nous avons tenté de faire une classification qui soit mutuellement exclusive. Cependant, il se pourrait que des homicides que nous avons classés dans les catégories «querelle», «défensif » ou «indéterminé » aient été provoqués par la jalousie, le désir de possession exclusive, le refus d'autonomie de la femme. Malgré les possibles biais liés aux données ou aux chercheurs, le tableau qui suit présente la distribution des 77 homicides conjugaux répertoriés sur l'île de Montréal entre 1954-1962 et 1985-1989. Sur les 77 homicides à caractère conjugal, nous en trouvons au moins 42 $(54,5 \%)$ qui relèvent de la possession.

La classification des homicides conjugaux commis sur l'île de Montréal

\begin{tabular}{lcccccc}
\hline & \multicolumn{2}{c}{$1954-1962$} & \multicolumn{2}{c}{$1985-1989$} & \multicolumn{2}{c}{ Ensemble } \\
\hline Sous-types & $\mathrm{N}$ & $\%$ & $\mathrm{~N}$ & $\%$ & Total & \% total \\
\hline Possession & 14 & 51,9 & 28 & 56 & 42 & 54.5 \\
Querelle & 6 & 22,2 & 12 & 24 & 18 & 23,4 \\
Libération & 2 & 7,4 & - & - & 2 & 2,6 \\
Euthanasic & - & - & 3 & 6 & 3 & 3,9 \\
Défensif & - & - & 2 & 4 & 2 & 2,6 \\
Instrumental & - & - & 1 & 2 & 1 & 1,3 \\
Accidentcl & - & - & 1 & 2 & 1 & 1,3 \\
Indéterminé & 5 & 18,5 & 3 & 6 & 8 & 10,4 \\
\hline TOTAL & 27 & 100 & 50 & 100 & 77 & 100 \\
\hline
\end{tabular}

La possession par l'homme est. de loin, le problème dominant dans l'homicide conjugal, et cela, aussi bien avant la Révolution tranquille qu'après. Examinons de plus près le cas suivant. 
Cas 1. Claudia ( 24 ans) et Roger ( 26 ans) sont mariés et ils ont un enfant. Le couple habite chez les parents du jeune homme. Les conjoints s'étaient divorcés deux ans après leur mariage et un an plus tard. ils se remariaient. Aux dires des témoins, les relations entre les époux semblaient bonnes jusqu'au moment où l'épouse a annoncé à son mari sa décision de divorcer à nouveau.

Selon la version du mari, le 12 février 1958. il demande à sa femme de ne pas le quitter. mais cette dernière refuse. Rejetant la perspective d'un nouveau divorce d'avec celle qu'il dit aimer, le mari descend au sous-sol durant la soirée dans l'intention d'aller réfléchir. Selon sa version des faits, la vue du fusil de chasse suspendu au mur lui a donné l'idée de supprimer sa femme. Il s'empare de l'arme, remonte à la chambre où sa femme est couchée, pointe son arme vers elle et presse la gâchette. La jeune femme, atteinte de deux balles, meurt sur le coup.

Cependant, la possession se combine aussi avec d'autres problèmes. Souvent, elle se pose dans un climat de mésentente qui alimente les altercations. Ainsi, dans au moins 9 cas sur les 42 où on retrouve la domination comme moteur principal du meurtre, les querelles étaient fréquentes dans la vie du couple.

L'homicide par possession est toujours commis par un homme et la victime est presque toujours une femme; la seule exception concerne les couples homosexuels. Au cours des deux périodes, 4 hommes qui voulaient quitter leur partenaire du même sexe ont été tués par lui.

Cas 2. Michel, un vendeur de 22 ans demeure avec Serge, un coiffeur de 22 ans : ils sont amants. Quelques semaines avant le meurtre, Michel (la victime) avait annoncé à Serge qu'il le quittait. Ce dernier a très mal réagi et à plusieurs reprises, il a menacé de se suicider.

Durant la soirée du mardi 2 février 1988, une violente querelle éclate entre les deux hommes. Au cours de l'altercation, vers onze heures du matin, Serge assomme Michel avec une massue et le projette ensuite dans le vide du haut du dix-neuvième étage. Quelques minutes après que la victime se soit écrasée. l'agresseur se lance dans le vide.

Le tableau fait aussi voir que $18(23,4 \%)$ homicides conjugaux sont sous la rubrique «querelle». Il s'agit d'homicides précédés d'une vive altercation au cours de laquelle les conjoints s'abreuvent d'injures et, quelquefois, échangent des coups. Une mésentente assez générale domine dans ces cas sans qu'il soit possible de découvrir une raison précise du conflit.

Actuellement, il n'est pas très compliqué pour les couples de se séparer, mais avant la loi de 1968, le divorce était exceptionnel. Certains hommes n'envisageaient pas d'autres solutions que le meurtre pour se 
libérer d'une personne avec qui, à leurs dires, la vie était insupportable. De Greeff (1942, p.140) qualifie ces homicides d'utilitaires, mais nous, pour notre part. les avons classés dans «libération". Ces meurtres de libération sont plutôt rares : les deux seuls cas repérés ont été commis entre 1954 et 1962.

Les trois homicides relevant de l'«euthanasie» ont été commis entre 1985 et 1989 par des hommes âgés qui ont tué leur femme dans l'intention de la délivrer de ses souffrances. Les meurtriers se sont d'ailleurs suicidés.

Les deux cas d’homicides «défensifs» ont été perpétrés durant les années 1985-1989. Ils sont le fait de femmes et, leur acte était une réponse à une attaque physique contre elles. Peut-être que d'autres homicides commis par des femmes auraient pu se retrouver sous cette rubrique, mais les circonstances et les motifs allégués n'étaient pas suffisamment précis pour ce faire.

On relève Également une affaire que l'on peut classer sous la désignation d' «instrumental " (un jeune homme attire sa femme sur le rebord d'un immeuble de cinq étages et la pousse dans le vide pour toucher l'assurance) et une autre qui paraît accidentelle (l'homme avait voulu soulager les souffrances de sa jeune compagne à la suite d'une chirurgie dentaire à l'aide d'une injection d'héroïne, il semble qu'il ne voulait pas la faire mourir).

D'autres homicides surviennent sans raison apparente: à tout le moins. nous n'en connaissons pas qui permettrait de les classer dans un des soustypes : huit font donc partie de la classification «indéterminé ». Dans certains cas, on pourrait toujours attribuer à l'aliénation mentale l'origine du drame, mais dans d'autres, il est impossible de savoir, surtout lorsque le meurtrier est trouvé mort aux côtés de la victime.

Compte tenu de l'importance de la possession comme motif de l'homicide conjugal, les sections suivantes seront consacrées à ce type d'homicide.

\section{Les conditions de l'homicide par possession}

Notre deuxième objectif consiste à identifier les conditions nécessaires à l'accomplissement de l'homicide conjugal dans la foulée des idées de Cohen et Felson (1979) puis de Gottfredson et Hirschi (1990). S'agissant de l'homicide par possession. les données nous permettent d'identifier cinq conditions nécessaires.

1) La remise en cause unilatérale du lien conjugal. Le meurtre commis par un homme qui ne veut pas perdre le contrôle sur sa compagne se produit quand la femme prend l'initiative de la séparation ou du divorce : quand elle annonce qu'elle rompt: lorsqu'elle veut quitter son amant: quand elle a déjà quitté son conjoint ou lorsqu'elle entretient une liaison. Dans le cas qui suit. la femme voulait divorcer d'avec son mari. 
Cas 3. Pauline ( 33 ans) et Romain ( 37 ans) sont mariés depuis trois ans. Leur relation n'étant pas très bonne, la femme avait décidé de quitter l'appartement.

Dix jours plus tard, soit le jeudi 29 mai 1986 vers dix-neuf heure trente. Pauline retourne voir son mari afin de lui parler de divorce. Dans sa version des faits. Romain dit avoir été surpris par une telle demande car il croyait que la séparation était temporaire et qu'elle allait plutôt les rapprocher. Selon sa version des faits, Romain tente de connaître les motifs de sa femme pour divorcer, celle-ci retuse de répondre. Tous deux haussent le ton : elle pour s'opposer à toutes explications et lui, pour connaître les raisons. Des paroles, la victime et le meurtrier passent aux actes et il y a bousculade (constatée par les policiers).

Le mari s'empare d'un couteau et il poignarde sa femme à plusieurs reprises au thorax pour ensuite l'étrangler avec une corde et l'achever à coups de martedu. Sans plus se préoccuper de sa femme, l'homme quitte l'appartement.

2) L'accessibilité de la future victime. Les études sur la violence conjugale montrent que plusieurs raisons retiennent les femmes de briser une relation violente dans laquelle elles se sentent comme prises au piège. Par exemple, l'homme ne tarit pas de promesses sur son éventuel changement et la femme garde l'espoir qu'il va cesser d'être violent: elle ne dispose pas des moyens financiers qui lui permettraient de partir ni d'appuis personnels susceptibles de l'aider; elle craint que la rupture incite son conjoint à se venger (Prairie et Langelier-Biron, 1985). Alors, elle ne quitte pas son conjoint ou elle ne va pas très loin, ou elle le revoit périodiquement. Si elle a réussi à s'éloigner, son «ex» établit des contacts avec ses relations, ses amis ou ses enfants pour la retracer et il la rejoint. D'ailleurs, Martin (1988) note que, souvent, la violence grave à l'endroit des femmes et leur meurtre surviennent après qu'elles aient divorcé ou qu'elles se soient séparées de leur partenaire. Dans le cas qui suit, le couple était divorcé depuis plusieurs années, mais l'homme et la femme vivaient à proximité l'un de l'autre.

Cas 4. Carla, une femme de 57 ans, a été mariée durant de nombreuses années à Yves, un plâtrier de 60 ans. L'homme est un alcoolique. Durant leur vie commune, la relation est fort turbulente, l'homme frappe souvent sa femme à coups de poings ou avec ce qui lui tombe sous la main. Il l'avait déjà trappée à coups de couteau et il avait même fait feu en sa direction. Les policiers ont souvent été appelés au domicile du couple pour cette violence du mari envers sa femme.

Le couple était divorcé depuis huit ans, mais la violence n'avait pas cessé pour autant. Carla habite le rez-de-chaussée avec son fils et Yves demeure 
au sous-sol. L'ex-mari cherche toujours son ex-femme et les deux se rencontrent de temps à autre. lorsque l'homme n'est pas en état d'ébriété. Au moment du meurtre. Yves était sous le coup d'une accusation pour voies de fait contre sa femme.

Le dimanche 24 mai 1987, vers vingt-deux heures quarante-cinq. des voisins entendent une querelle entre les protagonistes. Ils voient Carla sortir en trombe de son appartement et se précipiter vers sa voiture. Mais avant qu'elle ne l'atteigne, deux coups de feu claquent et une balle l'atteint au cou, lui sectionnant la carotide : Carla meurt sur le coup.

Au procès, la fille du couple a raconté que ce soir-là. son père cherchait son ex-femme et qu'il était fâché de ne pas la trouver. Il avait raconté à sa fille qu'il allait la tuer.

Certains des meurtres les plus révoltants sont ceux où le criminel pourchasse la femme qui a tout fait pour lui échapper. Par exemple, le cas de cette femme qui avait quitté son mari qui la battait et qui, un jour, l'avait blessée sérieusement en la poignardant. En faisant suivre les enfants qui demeuraient avec leur mère. l'homme avait réussi à retracer sa femme. Un samedi matin du mois de septembre 1985, muni d'un couteau de chasse. le mari fait irruption chez son épouse, se jette sur elle et la poignarde à douze reprises.

3) La vulnérabilité de la victime. Elle doit être privée des moyens de défense suffisants pour se protéger des attaques de son conjoint. Ici se pose le problème du rapport de forces : il est à l'avantage de la plupart des hommes qui possèdent une force musculaire supérieure à celle de leur femme. La plupart du temps. cette supériorité est accentuée par l'arme dont il s'est muni. Le fait que la femme soit isolée et qu'il n'y ait pas, au moment des faits, une personne qui aurait la force et le courage de $s$ 'interposer contribue à cette vulnérabilité. Dans certains cas où la femme a un nouveau conjoint ou un nouvel ami. celui-ci n'a pas le courage ou la force nécessaire pour jouer ce rôle de protecteur.

4) Le temps nécessaire pour que le problème conjugal arrive à maturité et entre dans sa phase critique. Dans tous les cas sur lesquels nous avons des informations. l'homicide conjugal-type est précédé d'une période plus ou moins longue d'incubation. De Greeff (1942) décrit le cheminement de l'homme qui voit la femme aimée se détacher de lui. Il commence par la revaloriser puis, généralement. il en vient à la dévaloriser, la réduisant peu à peu à un être porteur de tous les défauts. C'est progressivement qu'il s'achemine vers un dessein homicide. La femme qui ne fait rien pour neutraliser cette évolution, parce qu'elle ne l'a pas remarquée, parce qu'elle ne sait que faire ou parce qu'elle a essayé et n'a pas réussi, court le risque de se faire tuer. 
Les ruptures qui s'éternisent présentent le danger de donner au conjoint le temps d'amener son projet à maturité. Les femmes qui font alterner les ruptures et les réconciliations qui, un jour, partent et, un autre. reviennent, parce que l'homme fait de nombreuses promesses de changement, augmentent les risques que la rupture se termine mal.

5) La neutralisation de la prohibition du meurtre. L'homicide surtout celui d'un proche - est un acte sur lequel pèsent de formidables tabous. Notre surmoi nous l'interdit absolument, notre milieu social le condamne sans appel et nos tribunaux le punissent durement. Aussi faut-il surmonter maints obstacles intérieurs et extérieurs pour en arriver à pouvoir tuer.

Ces inhibitions sont affaiblies ou supprimées par quatre moyens : a) l'aveuglement de la passion: b) la rage ; c) l'accoutumance de la violence: d) l'alcool.

a) La plupart des meurtriers conjugaux sont habités par une passion dévorante. soit-disant amoureuse, qui les obnubile. La femme qui leur échappe devient tout pour eux. Devant la perspective de son départ, ils ont le sentiment de ne plus exister. Tout le sens de leur vie se déduit à cet amour rejeté, à cette passion exclusive. De Greeff (1942) rappelle que ce n'est pas tellement la douleur de l'amour perdu qui les fait agir, mais un sentiment d'outrage à leur valeur personnelle. Ils souffrent, mais dans leur volonté de domination. Leur passion est tellement forte que plus rien ne compte. Le futur meurtrier est devenu inintimidable : indifférent tant à son propre sort qu'aux années de pénitencier qui l'attendent. Dans plusieurs cas, le suicide est soit évoqué, soit tenté, soit exécuté. Parmi les 42 homicides conjugaux commis sous le signe de la possession, 14 meurtriers ont évoqué, tenté ou exécuté leur suicide : deux ont mentionné à une tierce personne qu'ils en avaient l'intention, deux ont tenté de le faire sans y réussir et dix sont passés à l'acte.

Cas 5. Jean ( 39 ans) et Louise (37 ans) sont mariés légalement et ils ont un fils dans l'adolescencc. Le frère de la victime habite à la résidence de là petite famille.

Durant la nuit du drame, le 11 janvier 1956, le frère et le fils de la victime sont tirés de leur sommeil par des cris provenant de la chambre du couple. Le frère s'y précipite et voit son beau-frère penché au-dessus de sa femme étendue sur le lit en train de lui assener des coups de rouleau à pâte à la tête. Il essaie en vain de l'arrêter. 11 appelle l'adolescent en pensant que ce dernier pourrait calmer son père. Mais le meurtrier les chasse en les menaçant. L'oncle conduit son neveu dans une autre pièce et revient pour porter secours à sa sceur. C'est là qu'il voit son beau-frère un couteau à la main. Il tente de le raisonner, mais le meuttrier part à sa poursuite et il doit se 
réfugier dans une autre pièce. Pendant ce temps, le fills était allé chercher ses grands-parents paternels qui habitaient le même immeuble.

Entre la sortie du fils et du beau-frère puis l'arrivée de la mère du meurtrier. le mari avait eu le temps de frapper sa femme de plusieurs coups de couteau et de lui trancher la gorge jusqu'à la colonne vertébrale. Cela fait, il avait tourné son arme contre lui-même et s'était enfoncé le couteau au cour. Avant de mourir, le meurtrier dit à sa mère qu'il avait été obligé de tuer sa femme parce qu'elle le trompait.

b) La fureur. l'état de rage extrême dans laquelle se met le meurtrier au moment de passer à l'acte l'aide aussi à sumonter les inhibitions qui l'empêchent d'agir. D'où la fréquence des altercations, des échanges verbaux et physiques de plus en plus violents qui précèdent immédiatement le meurtre.

Cas 6. Anna ( 50 ans) et Mario ( 52 ans) sont mariés depuis de nombreuses années. Depuis trois mois, le couple était séparé et la femme vivait chez son fils. Les époux devaient passer devant le tribunal le 9 février 1986 pour régulariser la séparation. Mais, le samedi 5 février, vers quatorze heures trente, Anna se présente à leur domicile pour signifier à son mari qu'il devait quitter le triplex car elle le gardait dans le partage des biens. Elle lui annonce Également qu'il devra lui payer une rente. Une violente discussion éclate et le mari, en colère. s'empare d'un couteau et frappe sa femme. Celle-ci réussit à le désarmer, mais l'homme prend un tisonnier et la transperce de part en part: ensuite, il tente sans succès de s'ouvrir les veines.

c) L'habitude de la violence. Les inhibitions contre le meurtre peuvent être progressivement levées par des comportements violents qui s'échelonnent au fill des ans. comme on l'a vu dans le cas 4 déjà rapporté plus haut.

d) L'alcool est également un déshinibiteur fort efficace, surtout dans le cadre d'une altercation. Les données sont insuffisantes pour imputer à l'alcool un rôle clé dans les homicides conjugaux. mais l'analyse a montré qu'au moment des faits. quelques meurtriers étaient sous l'effet de boissons alcoolisées ou de drogues. Cependant, plusieurs autres études recensées par Wilson et Hernstein (1985, p. 356) indiquent que l'alcool est très souvent présent dans les affaires de meurtre.

\section{Le déroulement de l'homicide conjugal}

Une explication complète d'un homicide conjugal devrait inclure son histoire ou. plus précisément, le récit des événements qui l'ont précédé. En effet, dans un conflit interpersonnel, la «cause» du geste de l'un est bien souvent un geste de l'autre. Considérons, par exemple. le cas suivant. Lynda, qui est divorcée d'avec Marcel, se retrouve au domicile de ce der- 
nier en train de discuter. La discussion s'envenime, on passe aux coups, Marcel tente d'étrangler son ex-femme (la police a ensuite trouvé des marques de doigts dans le cou de Lynda): la femme réussit alors à s'emparer d'un couteau et elle tue l'homme pour se défendre. Il est raisonnable de dire que la tentative faite par Marcel d'étrangler Lynda est la cause immédiate du coup de couteau porté par cette dernière.

Les homicides conjugaux motivés par la possession ne se déroulent pas tous de la même manière, mais certains éléments reviennent assez souvent pour qu'il soit possible de présenter un enchaînement des principaux événements. Voyons d'abord un cas où les principales étapes peuvent être décelées.

Cas 7. Josée (33 ans) et Carl (38 ans) sont mariés depuis quelques années et ils ont un fils de 5 ans. Depuis assez longtemps (selon la version de l'accusé et des voisins), la femme avait un amant. Elle délaisse son mari de plus en plus et ce dernier insiste pour que la vie reprenne comme auparavant. Josée lui fait comprendre qu'elle désire la séparation. Elle veut le quitter en emmenant leur enfant. Carl décide de se suicider, mais un de ses amis l'en dissuade. Selon la version du mari, le mardi 21 janvier 1986 (jour du drame), vers $17 \mathrm{~h} 30$, alors qu'ils sont dans leur appartement commun. Carl demande à Josée de rompre avec son amant. Celle-ci refuse, et le provoque en téléphonant à son amant. Carl se saisit du revolver chargé de 6 balles et il le dirige contre sa propre tempe tout en faisant signe à Josée de couper la communication. Cette dernière se moque de lui. Sans tenir compte de la présence de leur fils, Carl retourne l'arme contre Josée et lui tire les 6 balles en plein front.

Dans cette affaire, cinq étapes peuvent être repérées : 1- la femme a une liaison affichée et elle s'est détachée de son mari de qui elle veut se séparer : 2- le mari refuse la rupture et veut se suicider: 3 - elle le provoque en téléphonant à son amant; 4- le mari prend le revolver et il exige que sa femme coupe la communication mais celle-ci refuse : 5 - il tue sa femme.

Pour faire apparaitte la dynamique interne de l'enchaînement des faits durant l'homicide conjugal, il peut être utile d'en présenter le déroulement typique. Suivant la méthode du type-idéal de Weber, nous présentons une stylisation des caractéristiques du phénomène qui - souvent faute d'information - ne se retrouvent pas dans les affaires réelles mais dont on trouve les éléments éparpillés dans plusieurs cas différents. Il se déroule en cinq étapes.

Première étape : La rupture initiée. La fernme fait savoir qu'elle a décidé de rompre, ou elle se prépare à quitter le domicile conjugal, ou elle entame des procédures de divorce ou encore, le conjoint découvre qu'elle entretient une liaison. 
Deuxième étape : La mort annoncée. Le conjoint juge la rupture inacceptable. Pour lui, la femme «n'a pas le droit de lui faire ça». Il fait alterner promesses et accusations. Il lance des ultimatums. Il menace de se suicider, puis il jure qu'il la tuera. Il se procure une arme.

Troisième étape : Le défi. La femme pose des gestes visant à montrer que sa décision est irrévocable. Elle confirme que la rupture est définitive. Elle tait ses valises. Elle demande à son avocat d'entamer les procédures de divorce. Elle affiche sa liaison. Elle téléphone à son amant sous les yeux de son mari. Elle le fait venir au domicile conjugal. Pendant ce temps, le contact entre les conjoints n'est pas complètement rompu: soit ils vivent encore sous le même toit ou à proximite; soit ils continuent de se revoir; soit le conjoint découvre la trace de la femme.

Quatrième étape : L'altercation. Les partenaires s'échangent des insultes; ils ressassent de vieux griefs: ils se disputent avec acrimonie sur le partage des biens; ils en viennent aux coups.

Cinquième étape : La mise à mort. L'homme est arrivé au point où la colère et le désespoir le poussent au meurtre. Dans un paroxysme de rage, il terrasse la femme et $s$ 'acharne sur elle : il la frappe de multiples coups de couteau : il lui fracasse la tête sur un mur; il la bat à mort...

$* * * *$

Que le lecteur nous permette de conclure par un bref résumé suivi d'une remarque méthodologique.

Dans cet article, nous nous proposions moins de découvrir les causes de l'homicide conjugal que d'en pénétrer l'intelligibilité. La possession entendue comme la volonté masculine de contrôle exclusif sur l'être aimé - est, de loin, la raison qui pousse le plus fréquemment un homme à tuer la personne avec qui il partage sa vie, généralement une femme et dans quelques cas un amant du même sexe : plus de la moitié des homicides conjugaux commis sur l'île de Montréal sont motivés par cette jalousie dominatrice et possessive. Il ne suffit cependant pas qu'un homme soit habité par cette passion pour qu'il en arrive à tuer sa compagne. Un conflit conjugal de ce type n'a de chances de fïnir tragiquement que si cinq conditions sont réunies: 1) La femme remet en cause unilatéralement le lien conjugal : 2) l'homme peut atteindre physiquement la femme: 3 ) le rapport de forces doit avantager l'agresseur, ce qui met la temme en état de vulnérabilité : 4) le temps doit faire son auvre pour que le projet criminel arrive à maturité : 5) le meurtrier doit réussir à surmonter les inhibitions qui empêchent la quasi-totalité des hommes d'arriver à cette extrémité.

Dans son déroulement typique, l'homicide par possession débute par une volonté de rupture initiée par la femme a laquelle le conjoint oppose une tïn de non-recevoir assortie de menaces. La femme maintient malgré 
tout sa résolution, ce qui fait déboucher le conflit sur une altercation qui débouche sur la mort.

Il faut aussi savoir que l'homicide conjugal est parfois annoncé à l'avance : le futur meurtrier claironne sur tous les toits qu'il tuera sa femme (voir le cas 4). De tels propos doivent être pris au sérieux. Les intentions suicidaires et les tentatives de suicide sont aussi des signaux très graves. Ils signifient que l'homme est prêt à tout. Il suffit alors de très peu de choses pour qu'il tourne sa violence sur l'autre. Parmi les autres faits qui doivent être considérés comme des clignotants, signalons : l'achat d'une arme à feu par le conjoint, le fait qu'il ait un passé violent, les altercations qui deviennent de plus en plus en plus vives et l'escalade de la violence. Si ces signaux apparaissent dans la situation du processus de rupture refusée par l'homme, l'affaire risque de finir tragiquement.

Soulignons aussi que les femmes tuent rarement celui avec qui elles ont établi une relation de confiance. Lorsqu'elles le suppriment, c'est soit pour se défendre contre des attaques physiques de sa part, soit au cours d'une violente querelle. Ce qui n'exclut pas l'autodéfense. mais les données étaient insuffisantes pour les classer comme tel.

Pour finir, quelques remarques sur la méthode. Il se pourrait que notre approche de l'homicide conjugal s'applique aussi à l'analyse de plusiers autres types de délits. Dans la tradition positiviste, on explique le crime par des causes exogènes : le chômage, la désorganisation sociale... Pour échapper au réductionnisme d'une telle démarche, il nous semble préférable de commencer par pénétrer la dynamique interne du crime que l'on veut expliquer. On commence par rendre compte du phénomène en saisissant le rôle de ses éléments constitutifs. Dans le cas de l'homicide, ces éléments sont : le criminel, sa victime, le conflit qui les oppose, le lien qui les unit. la situation dans laquelle ils se trouvent, les armes dont ils disposent, les objectifs qu'ils poursuivent... Une telle démarche exige du chercheur qu'il reste collé aux faits, qu'il tente d'en décoder le sens et qu'il reconstitue la chaîne des événements. Cela pourrait vouloir dire que l'analyse d'un type particulier de crimes devrait commencer par une démarche en quatre étapes : identification d'un pattern criminel homogène (en l'occurrence. l'homicide conjugal par possession); étude des raisons, des fins et des motivations des acteurs; énumération des conditions nécessaires du crime : récit du déroulement typique des faits. Ce n'est qu'ensuite que l'on pourrait s'interroger sur les facteurs externes qui auraient pu jouer sur l'un ou l'autre des éléments constitutifs du crime. 


\section{BIBLIOGRAPHIE}

BERK, R. A., BERK, F., LOSEKE, D. R. et RAUMA, D. (1983), « Mutual Combat and Other Family Violence Mythsw, in D. Finkelhor, R.J. Gelles, G.T. Hotaling, M.A. Straus (dir.). The Dark Side of Fumilies, Beverly Hills, Sage Publications.

BLAU, J. R. et BLAU. P. M. (1982), "The Cost of Inequality : Metropolitan Structure and Violent Crime», American Sociological Review, vol. 47, pp. 114-129.

BOUDON, R. (1992), "L'action sociale», in R. Boudon (dir.), Manuel de sociologie. Paris, Presses universitaires de France.

CHIMBOS, P. D. (1978), Marital Violence: A Study of Interspouse Homicide, San Francisco, R\&E Research Associates Inc.

COHEN, L. et FELSON, M. (1979), "Social Change and Crime Rate Trends: A Routine Activity Approach ", American Sociological Review, n 44, pp.588-608.

CORNISH, D. B. et CLARKE, R. V. (1986), The Reasoning Criminal, New York, Springer-Verlag.

CUSSON, M. (1981), Délinquants pourquoi?, Montréal, Hurtubise.

CUSSON, M. (1990), Croissance et décroissance du crime, Paris, Presses universitaires de France.

CUSSON, M. et CORDEAU, G. (1993), «Le crime du point de vue de l'analyse stratégique», in D. Szabo et M. Le Blanc (dir.), La Criminologie empirique au Québec, Montréal, Presses de l'Université de Montréal.

DALY, M., WILSON, M. et WEGHORST, S. J. (1982), "Male Sexual Jealousy", Ethology and Sociobiology, vol. 3, pp. 11-27.

DALY, M. et WILSON, M. (1988), Homicide, New York, Aldine De Gruyter.

DALY, M. et WILSON, M. (1988a), «Evolutionary Social Psychology and Family Homicide ", Science, vol. 242, pp. 519-524.

DE GREEFF, É. (1942), Amour et crimes d'amour, Bruxelles, C. Dessert (édition 1973).

DOBASH, E. R et DOBASH, R. P. (1979), Violence Against Wives. A Case Against the Patriarchy, New York, The Free Press.

DOBASH, E. R et DOBASH, R. P. (1988), «Research as Social Action. The Struggle for Battered Women», in Kersti Yllö, Michele Bograd (dir.), Feminist Perspectives on Wife Abuse, Newbury Park, Sage Publications, pp. 5I-73.

DOBASH, R., DOBASH, E., WILSON, M. et DALY, M. (1992), «The Myth of Sexual Symmetry in Marital Violence ", Social Problems, vol. 39, $\mathrm{n}^{\circ} \mathrm{I}$, pp. 71-89.

FELSON, R. B. (1993), Crime and Everyday Life. Insight and Implications for Society, Thousand Oaks, Californic, Pine Forge Press, Sage Publications.

GARTNER, R. (1990), "The Victims of Homicide : A Temporal and Cross-national Comparison *, American Sociological Review, vol. 55, pp. 92-106.

GOETTING, A. (1989), «Patterns of Marital Homicide : A Comparison of Husbands and Wives», Journal of Comparative Family Studies, vol. XX, $\mathrm{n}^{\prime \prime} 3$, pp. 341-354.

GOTTFREDSON, M. R. et HIRSCHI, T. (1990), A General Theory of Crime, Stanford, Stanford University Press.

GOUVERNEMENT DU QUÉBEC (1993). Statistiques 92. Violence conjugale. Ministère de la sécurité publique, Direction des altaires policières. 
GRENIER, S. (1993), L'évolution des catégories d'homicides au Québec de 1954 à 1989, mémoire de maîtrise, Montréal, École de criminologie, Université de Montréal.

GUILlaIS, J. (1986), La Chair de l'autre, Paris, Olivier Orban.

GUTTMACHER, M. S. (1955), «Criminal Responsability in Certain Homicide Cases Involving Family Members ", in P. H. Hoch et J. Zubin (dir.), Psychiatry and the Law, New York, Grune \& Stratton.

LAROUCHE, G. (1987), Agir contre la violence, Montréal, Les Éditions de la Pleine Lune.

LUCKENBILL, D. (1977), "Criminal Homicide as a Situated Tansaction ", Social Problems, vol. $24 \mathrm{n}^{\circ}$ 2, pp. 176-186.

MARTIN, D. (1976), Batterd Wives, San Francisco, Glide.

MARTIN, M. (1988), «Battered Women", in Nancy Hutchings, M.S.W. The Violent Family. Victimization of Women, Children. and Elders, New York, Human Sciences Press, Inc. pp. 62-88.

MESSNER, S. F. (1982), « Poverty, Inequality, and the Urban Homicide Rate», Criminology, vol. 20, $\mathrm{n}^{\circ} 1$, pp. 103-114.

NETTLER, G. (1982), Explaining Criminals, Cincinnati, Anderson Publishing.

PARKER, R. N. et TOTH, A. M. (1990), «Family, Intimacy, and Homicide : A MacroSocial Approach ", Violence and Victims, vol. 5, $\mathrm{n}^{\circ} 3, \mathrm{pp} .195-209$.

POLK, K. et RANSON, D. (1991), "The role of Gender in Intimate Homicide", Australia and New Zeland Journal of Criminology, vol. 24, pp. 15-24.

PRAIRIE, J. et LANGELIER-BIRON, L. (1985), Violence conjugale : processus d'arrêt, Montréal, Université de Montréal, Centre international de criminologie comparée.

PROAL, L. (1900), Le Crime et le suicide passionnels, Paris, Félix Alcan.

RABINOWICZ, L. (1931), Le Crime passionnel, Paris, Librairie des sciences politiques.

SAUNDERS, D. G. (1988), "Wife Abuse, Husband Abuse, or Mutual Combat?" in Kersti Yllö, Michele Bograd (dir.), Feminist Perspectives on Wife Abuse, Newbury Park, Sage Publications, pp. 90-113.

SILVERMAN, R. et KENNEDY, L. (1993), Deadly Deeds. Murder in Canada, Scarborough, Ontario, Nelson Canada.

STATISTIQUE CANADA (1992), L'homicide au Canada 1991, Ottawa, Juristat, Centre canadien de la statistique, ministère des Approvisionnements et Services Canada, vol. 12, n" 18.

STOUT, K. D. (1989), «"Intimate Femicide" : Effect of Legislation and Social Services $»$, Affilia, vol. 4, no 2 , pp. 21-30.

WILLIAMS, K. R. (1984), «Economic Sources of Homicide, Reestimating the Effects of Poverty and Inequality ", American Sociological Review, vol. 49, pp. 283-289.

WILSON, A. V. (1993), Homicide: The Victim/Offender Connection, Cincinnati, Anderson Publishing Co.

WILSON, M. et DALY, M. (1992), «Who Kills Whom in Spouse Killings? The Exceptional Sex Ratio of Spousal Homicides in the United States», Criminology, vol. $30, \mathrm{n}^{\circ} 2$, pp. 189-215.

WILSON, J. Q. eI HERNSTEIN, R. J. (1985). Crime and Human Nature, New York, Simon and Schuster. 
ZHAN, M. A. (1975), "The Fenale Homicide Victim», Criminology, vol. 13, $\mathrm{n}^{\circ} 3$, pp. 400-415.

ZIMRING, F. E.. MUKHERJEE, K., et VAN WINKLE, B. (1983), «Intimate Violence : A Study of Intersexual Honicide in Chicago ", University of Chicago Law Review, vol. 50, np. 910-930. 